\title{
Regulation and Potential Biological Role of Fibroblast Growth Factor 21 in Chronic Kidney Disease
}

\author{
Xue Zhou' ${ }^{1}$ Yuefeng Zhang ${ }^{1}$ and Ning Wang ${ }^{2 *}$ \\ ${ }^{1}$ Department of Nephrology, Tianjin Haihe Hospital, Tianjin, China, ${ }^{2}$ Tianjin Third Central Hospital, Tianjin, China
}

\section{OPEN ACCESS}

Edited by:

Belisario Enrique Fernandez, University Institute of Health Sciences,

Argentina

Reviewed by:

Carolina Susana Cerrudo,

Universidad Nacional de Quilmes

(UNQ), Argentina

Ana Maria Balaszczuk,

University of Buenos Aires, Argentina

Elsa Zotta,

University of Buenos Aires, Argentina

${ }^{*}$ Correspondence:

Ning Wang

wn20121004@126.com

Specialty section:

This article was submitted to Renal and Epithelial Physiology,

a section of the journal

Frontiers in Physiology

Received: 26 August 2021 Accepted: 15 September 2021

Published: 05 October 2021

Citation:

Zhou $X$, Zhang $Y$ and Wang N (2021) Regulation and Potential Biological Role of Fibroblast Growth Factor 21 in Chronic Kidney Disease.

Front. Physiol. 12:764503.

doi: 10.3389/fphys.2021.764503
Chronic kidney disease (CKD) is an incurable progressive disease with the progressive impairment of kidney function, which can accelerate the progression of cardiovascular disease, increase the risk of infection, and lead to related complications such as anemia and bone disease. CKD is to a great extent preventable and treatable, and it is particularly important to improve the early diagnosis, strengthen the research underlying the mechanism of disease occurrence and development, and innovate new intervention measures. Fibroblast growth factor 21 (FGF21) belongs to one of members of endocrine FGF subfamily with evolutionarily conserved functions and performs a vital role in the regulation of energy balance and adipose metabolism. FGF21 needs to rely on $\beta$-Klotho protein to specifically bind to FGF receptor (FGFR), which activates the FGF21 signaling exerting the biological function. FGF21 is deemed as an important regulatory factor extensively modulating many cellular functions under physiologic and pathologic conditions. Although the metabolic effect of FGF21 has been extensively studied, its potential biological role in the kidney has not been generally investigated. In this review, we summarize the biological characteristics, regulation and biological function of FGF21 based on the current studies, and briefly discuss the potential relationship with chronic kidney disease.

Keywords: fibroblast growth factor 21, biomarker, chronic kidney disease, diabetic nephropathy, cardiovascular disease

\section{INTRODUCTION}

Chronic kidney disease (CKD) is a progressive disease characterized by high morbidity and mortality, which is characterized by the changes in the structure and function of the kidney owing to various reasons (Kalantar-Zadeh et al., 2021). With the high and still increasing global burden of CKD, approximately 10\% of adults are affected by CKD (GBD Chronic Kidney Disease Collaboration, 2020). Overall speaking, the incidence of CKD increases with age, especially in patients with obesity, diabetes and hypertension (Silverwood et al., 2013;

Abbreviations: CAG, coronary angiography; CIN, contrast-induced nephropathy; CKD, chronic kidney disease; eGFR, estimated glomerular filtration rate; ESRD, end-stage renal disease; FGF21, fibroblast growth factor 21; FGFR, fibroblast growth factor receptor; GLP-1, glucagon-like peptide-1; NAFLD, non-alcoholic fatty liver disease; NASH, non-alcoholic steatohepatitis; PPAR $\alpha$, peroxisome proliferator-activated receptor- $\alpha$; ROS, reactive oxygen species; SGLT2, sodium glucose cotransporter 2; UGE, urinary glucose excretion. 
Cockwell and Fisher, 2020). CKD can accelerate the progression of cardiovascular disease, increase the risk of infection, and lead to anemia and bone disease, as well as other complications that increase the risk of premature death. Since the progression of CKD usually takes several years and the symptoms remain not obvious, early detection of the disease is particularly important. Non-drug strategies such as diet and lifestyle adjustments, and specific drug interventions can be adopted to improve the clinical outcomes of the patients with CKD.

In the past few decades, some new biomarkers have been discovered, which contributes to recognizing renal function impairment earlier. Fibroblast growth factor 21 (FGF21), one of the emerging biomarkers, has been associated with CKD (Kondo et al., 2020). Studies have verified that serum FGF21 levels in patients with CKD increase progressively and reach 20 times over the normal range (Hindricks et al., 2014). Moreover, in type 2 diabetes patients, the level of serum FGF21 is significantly linked to the occurrence of nephropathy, proteinuria, and the progression of end-stage renal disease (ESRD) (Jian et al., 2012; Lee et al., 2015). This article will review the biological characteristics, regulation and biological function of FGF21 in the onset and development of CKD, and finally evaluate the underlying role as a therapeutic target.

\section{BIOLOGICAL CHARACTERISTICS OF FIBROBLAST GROWTH FACTOR 21}

\section{Molecular Structure of Fibroblast Growth Factor 21}

As a member of the superfamily of fibroblast growth factors, FGF21 is composed of similar structure containing 150-300 amino acids (Itoh and Ornitz, 2011). FGF21, initially discovered in 2000, was most similar to FGF19 with approximately $35 \%$ similarity in the members of human FGFs (Nishimura et al., 2000). FGF21 precursor is composed of 209 amino acids encoded by 4 exons. Next, undergoing cleavage of a signal peptide, FGF21 precursor is converted into mature FGF21 containing 181 amino acids with molecular weight of approximately $20 \mathrm{kDa}$ (Zhang et al., 2015).

\section{Fibroblast Growth Factor 21 Related Receptors}

Studies have found that FGF exerts biological functions by binding to FGF receptors (FGFRs) belonging to tyrosine kinase receptors. However, the endocrine FGF (FGF19, FGF21, and FGF23) has a low affinity with FGFRs, and requires the participation of specific transmembrane glycoproteins ( $\alpha$ or $\beta$-klotho) in the target organs. Klotho protein is an important part of the endocrine FGF receptor complex and is indispensable to the high-affinity binding between FGF and FGFR (FernandesFreitas and Owen, 2015; Kuro-O, 2019b). In recent years, a growing body of researches have revealed that the FGFKlotho complex also participates in the pathophysiology of some diseases, involving CKD, diabetes, arteriosclerosis and cancer (Kuro-o, 2012). Consequently, through the thorough research on the FGF-Klotho-FGFR complex, the development of drugs targeting the FGF-Klotho endocrine axis may bring clinical benefits in multiple systems.

The specific binding between FGF21 and corresponding FGFR relies on $\beta$-Klotho protein (Ding et al., 2012). $\beta$-Klotho protein preferentially combines with FGFR for inhibiting paracrine FGFs signaling (Goetz et al., 2007), which helps endocrine FGFs to specifically bind to FGFRs in target cells avoiding the interference of paracrine FGFs. FGF signaling pathway with abundant structural information extensively modulates distinct biological process in development, tissue homeostasis and metabolism (Goetz and Mohammadi, 2013). In liver, FGF21 participates in regulating carbohydrate and fatty acid metabolism, including fatty acid oxidation and gluconeogenesis (Potthoff et al., 2009). In addition, FGF21 increases glucose uptake and lipolysis through converting white fat into brown fat for increasing energy metabolism (Emanuelli et al., 2014).

\section{Regulation and Function of Fibroblast Growth Factor 21}

FGF21, a hormone mainly produced in liver, is induced directly by peroxisome proliferator-activated receptor- $\alpha$ (PPAR $\alpha)$ (Inagaki et al., 2007). In the fasting or starvation state, PPAR mediates the increase in the expression of FGF21 in the liver, leading to gluconeogenesis, fatty acid oxidation and ketone body production as an adaptive response to hunger (Woo et al., 2013). PPAR $\alpha$ agonists, such as bezafibrate and a novel drug MHY2013, can significantly increase the expression of FGF21 for alleviating obesity-induced insulin resistance, dyslipidemia and hepatic steatosis (An et al., 2017). The regulatory process of FGF21 expression can be exhibited utilizing a diagram (Figure 1).

Recently, the biological characteristics of FGF have been extensively studied. The endocrine FGFs play multifaceted roles in the treatment of many chronic diseases involving in kidney disease, cardiovascular disease, obesity, type 2 diabetes, and cancer (Degirolamo et al., 2016). The abnormal signal of FGF is significantly linked to the development of cancer and metabolic diseases. The function of FGF21 was originally discovered in 2005 when looking for new drugs for the treatment of diabetes. FGF21 metabolic axes extensively participates in regulating the metabolic homeostasis (Li, 2019). FGF21 can improve glucose uptake by fibroblasts and adipocytes showing the characteristics of effective treatment of diabetes (Kharitonenkov et al., 2005). At present, FGF21, deemed as a metabolism-related hormone, is an emerging therapeutic target for metabolic diseases (Angelin et al., 2012; Lancha et al., 2012; Reitman, 2013). Furthermore, FGF21 can improve tissue damage caused by the harmful effects of metabolic abnormalities, including oxidative, inflammatory, and immune stress state (Luo et al., 2017). Consequently, some targeting FGF21 analogs have been developed for the treatment of metabolic disorders (Zhang and Li, 2015).

The latest research evidence confirms that FGF21 can improve metabolic status with anti-fibrotic effects and has the potential treatment for non-alcoholic steatohepatitis (NASH) (Harrison et al., 2021). A new type of long-acting FGF21 (LAPS-FGF21) has been developed for potential therapeutic effects on obesity. 


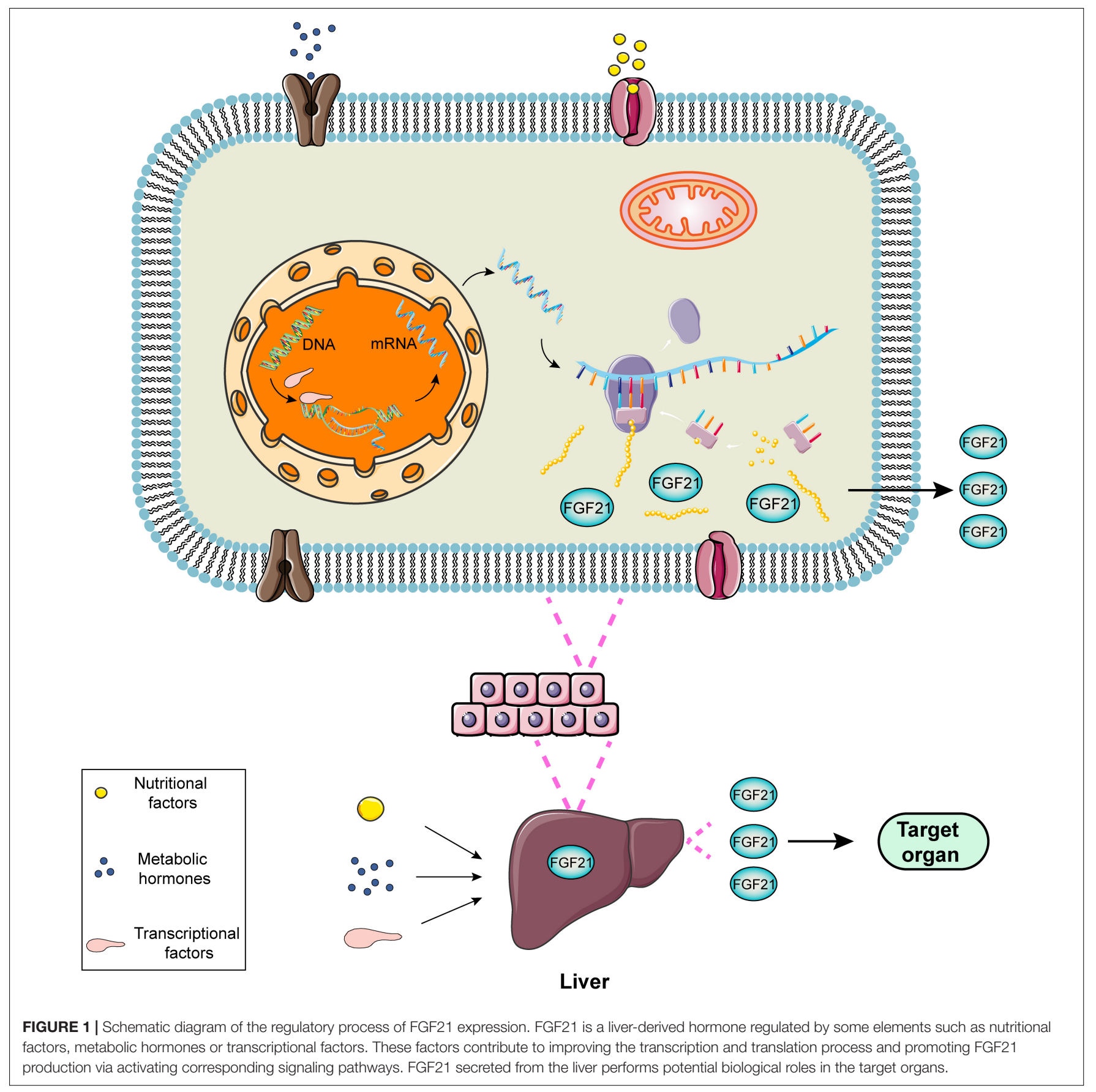

LAPS-FGF21 is chemically coupled with human IgG4 Fc fragment for a longer half-life in the serum, which can effectively reduce body weight and improve glucose tolerance in a dosedependent manner at the same time (Kim et al., 2021). FGF21 is highly expressed in the exocrine glands of the pancreas, the mechanism of which requires the FGFR-Klotho signaling transduction (Coate et al., 2017). Under physiological conditions, acute exercise can upregulate the expression level of FGF21 in skeletal muscle (Di Raimondo et al., 2016; Tanimura et al., 2016). At the same time, FGF21 has a higher level in patients with mitochondrial diseases affecting skeletal muscle, which can be used as a biomarker for mitochondrial respiratory chain defects in muscles (Suomalainen et al., 2011; Suomalainen, 2013; Nohara et al., 2019).

FGF21 is released by cardiomyocytes for avoiding hypertrophy, and also participates in regulating the expression of antioxidant pathway genes for reducing reactive oxygen species (ROS) mediated oxidative stress in cardiomyocytes, and acting as an antioxidant factor in the heart to control inflammation and cardiac hypertrophy (Planavila et al., 2013, 2015). Moreover, FGF21 can prevent atherosclerosis by regulating the interconnection among adipose tissue, liver and 
blood vessels (Lin et al., 2015), and activating the angiotensin converting enzyme 2-angiotensin axis for preventing angiotensin II-induced hypertension and vascular damage (Pan et al., 2018). In addition, FGF21 can also play a therapeutic effect on atherosclerosis through the NF- $\kappa \mathrm{B}$ pathway (Zhang et al., 2018). During cardiac remodeling in uremic cardiomyopathy, the effect of increased FGF21 expression on cardioprotective is needed to be further clarified (Suassuna et al., 2020). FGF21 partially ameliorates hyperglycemia by reducing renal glucose reabsorption based on the sodium glucose cotransporter 2 (SGLT2) pathway (Li et al., 2018b).

Since FGF21 is mainly excreted by the kidney (He et al., 2018), it can be predicted by the relative change of creatinine. The estimated glomerular filtration rate (eGFR) is a strong independent negative predictor of FGF21. Synergistic therapy of glucagon-like peptide-1 (GLP-1) and glucagon receptors can upregulate the expression of FGF21 and abate renal insufficiency induced by diabetes (Patel et al., 2018). Furthermore, one study has found that FGF21 has a protective effect on kidney against low protein diet-induced renal damage and inflammation (Fang et al., 2021). It has been reported that the level of circulating FGF21 is independently correlated with the occurrence of contrast-induced nephropathy (CIN) and corresponding kidney injury in patients receiving coronary angiography (CAG) (Wu et al., 2018).

\section{FIBROBLAST GROWTH FACTOR 21 AND DIABETIC NEPHROPATHY}

FGF21 is closely related to metabolic disorders including diabetes. In order to clarify the relationship between FGF21 and blood glucose, the results of a cohort study demonstrated that the level of FGF21 in the plasma of diabetic patients significantly increased, and was identified as an independent predictor of type 2 diabetes predicting the development of diabetes (Chen et al., 2011). A study has found that the high serum FGF21 level is correlated with low urinary glucose excretion (UGE) in type 2 diabetes patients (Zhang et al., 2021). Moreover, a metaanalysis found that FGF21 level in the plasma of type 2 diabetes patients significantly increased compared with the control group, which was affected by the variables of body mass index (BMI), total cholesterol and triglycerides (Wang et al., 2019). There is evidence existing to support that genetic variation in the FGF21 gene region is related to the renal function of type 2 diabetes patients and affects the eGFR of diabetic patients (Yu et al., 2019).

Studies have found that FGF21 levels can be used as a biomarker related to the prognosis of patients with diabetic nephropathy (El-Saeed and El-Mohasseb, 2017; Chang et al., 2021). Serum FGF21 levels are closely associated with early diabetic nephropathy in high-risk groups of type 2 diabetes patients, especially the circulating FGF21 value increasing more than $181 \mathrm{pg} / \mathrm{mL}$, so that effectively targeting FGF21 therapy may contribute to early detection and prevention of diabetic microvessels complication (Esteghamati et al., 2017). A crosssectional study also confirmed that elevated serum FGF21 level may be a useful biomarker for predicting the progression of kidney disease, especially in the early stage of diabetic nephropathy. Additionally, a recombinant human FGF21, PEGylated rhFGF21 (PEG-rhFGF21), has been developed for the treatment effect on diabetic nephropathy in diet induced obesity animal model (Zhao et al., 2017).

Insulin resistance is a pivotal process in the occurrence and development of diabetic nephropathy. Studies have found that alprostadil (prostaglandin E1) can reduce the insulin resistance via the autophagy-dependent FGF21 pathway for preventing the progression of diabetic nephropathy (Wei et al., 2018). FGF21 can negatively regulate TGF- $\beta$-p53-Smad2/3-mediated epithelial-to-mesenchymal transition by activating AKT for reducing diabetes-induced renal fibrosis (Lin et al., 2020). Based on the $\mathrm{db} / \mathrm{db}$ mouse model research, targeting FGF21 treatment could function as a potential therapeutic strategy in type 2 diabetic nephropathy for significantly down-regulating FGF21 receptor components, activating ERK phosphorylation, reducing the excretion of urinary albumin and mesangial expansion, inhibiting the synthesis of pro-fibrotic molecules, and improving renal lipid metabolism and oxidative stress damage (Kim et al., 2013). FGF21 protects kidney from damage by alleviating renal lipid accumulation and inhibiting inflammation, and fibrosis effects in diabetic nephropathy (Zhang et al., 2013). Through upregulating the expression of FGF21 and activating Akt2/GSK$3 \beta /$ Fyn/Nrf2 antioxidants and the AMPK pathway, fenofibrate can exert a role in preventing diabetic nephropathy in the patients with type 1 diabetes (Cheng et al., 2020). In addition, activation of FGF21 pathway may correlate with the effect of SGLT2 inhibitors on protecting the renal function in type 2 diabetes and delaying progression of CKD (Packer, 2020).

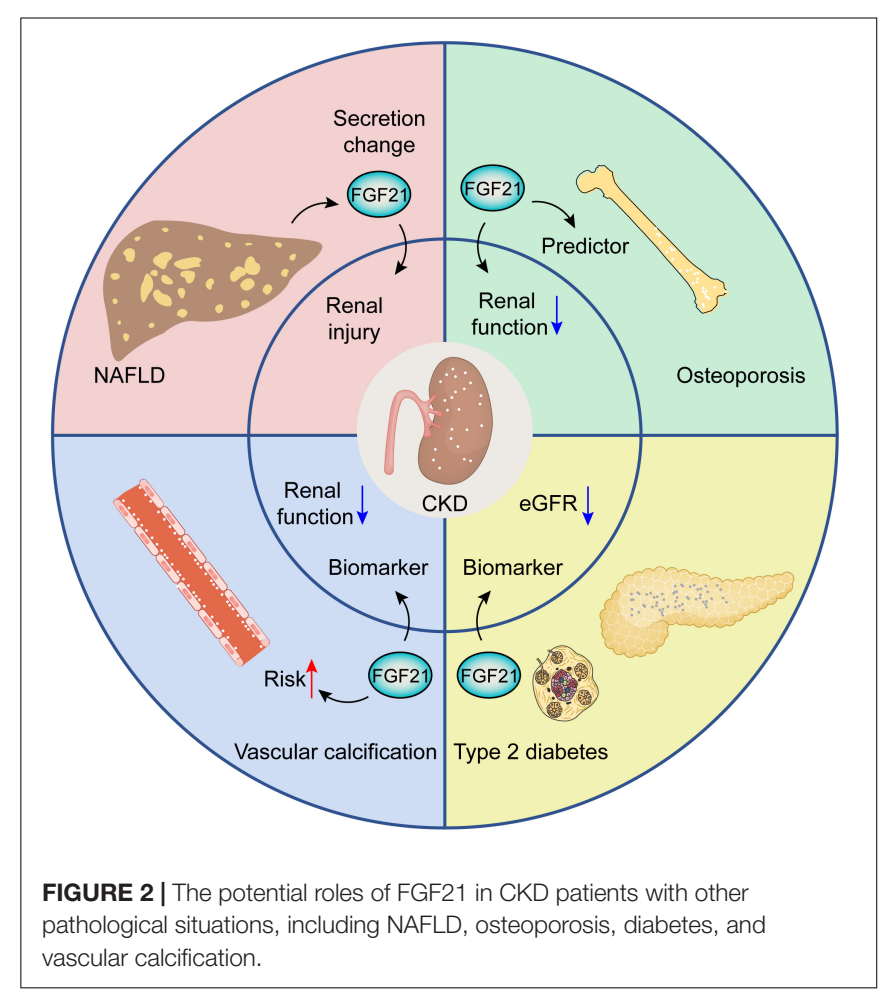




\section{FIBROBLAST GROWTH FACTOR 21 AND CHRONIC KIDNEY DISEASE}

In 2009, Stein et al. (2009) reported the correlation between FGF21 and kidney disease for the first time, and found that the circulating FGF21 level of chronic hemodialysis patients increased by 15 times compared with the control group based on Caucasian population in a study from Germany. Subsequently, some studies have found an 8-fold increase of FGF21 in peritoneal dialysis patients compared to normal subjects according to a study from Korean (Han et al., 2010). In clinical practice, multiple studies have shown that the level of serum FGF21 is correlated with renal function of the patients with CKD (Crasto et al., 2012). Furthermore, the elevated plasma FGF21 level in the Chinese population significantly correlated with the state of CKD progression, and is independently linked to renal function and poor blood lipid levels (Lin et al., 2011).

The determination of FGF21 may help evaluate CKD and its complications, which is expected to become a relevant biomarker of CKD (Kuro-O, 2019a; Yamamoto et al., 2020). Serum FGF21 levels in CKD patients are positively associated with oxidative stress, and negatively associated with eGFR based on a crosssectional study from Mexico (Ángel et al., 2021). The increase in FGF21 concentration in CKD patients may be related to the metabolism of lipids and carbohydrates, and FGF21 levels in CKD patients can be reduced through hemodialysis and transplantation from a Poland study (Marchelek-Myśliwiec et al., 2019). In peritoneal dialysis patients, FGF21 can be used as a hormone signal exerting a protective role in maintaining blood glucose homeostasis and preventing potential insulin resistance (González et al., 2016).

There exert potential roles of FGF21 in CKD patients with other pathological situations (Figure 2). Growing evidence points to the potential interplay between non-alcoholic fatty liver disease (NAFLD) and CKD, the patients with NAFLD can result in renal injury by means of the alterations of FGF21 secretion (Musso et al., 2015). Similar findings have uncovered that FGF21 can serve as a biomarker for CKD progression and is

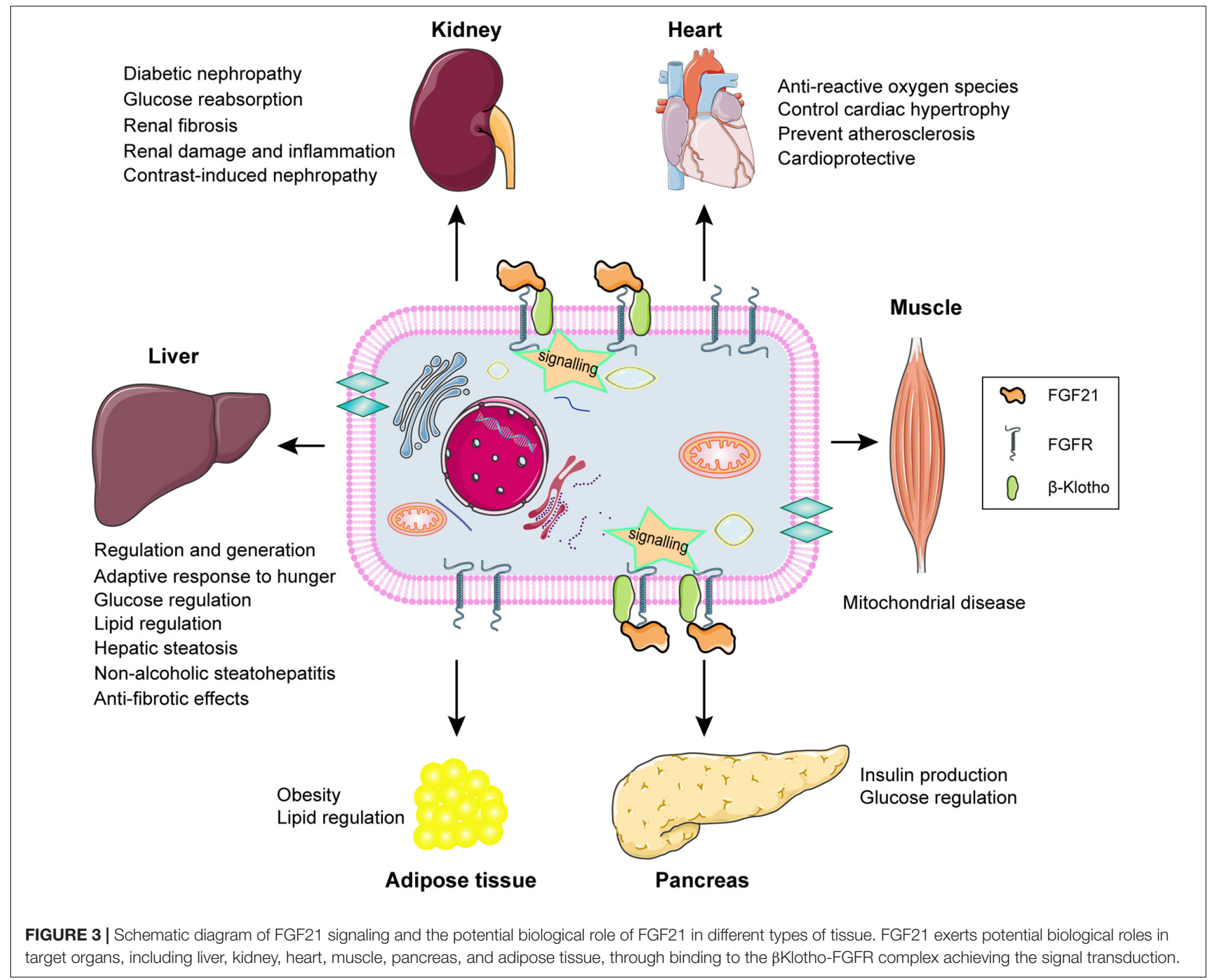


associated with an increased risk of vascular calcification in CKD patients (Kuro-O, 2019a). FGF21 can be deemed as a sensitive predictor associated with osteoporosis in hemodialysis patients with worse renal function (Zhu et al., 2021). Serum FGF21 has been confirmed as a biomarker for predicting rapid progression of CKD patients with type 2 diabetes through eGFR decline (Looker et al., 2015).

Some studies have shown that acute kidney injury may accelerate the progression of CKD. Therefore, prevention of acute kidney injury is an important part of the treatment for CKD. In a mouse model of acute kidney injury induced by cisplatin, the application of recombinant FGF21 can remarkably downregulate the relevant protein levels of kidney injury ( $\mathrm{Li}$ et al., 2018a; Chen et al., 2020). Additionally, another study demonstrates that the protective role of FGF21 in kidney injury can be induced by vascular calcification (Shi et al., 2018). Higher circulating FGF21 levels in patients with ESRD, but not with cardiovascular events, are associated with high mortality, which indicates that circulating FGF21 level can be used as a predictor for the prognosis of patients with CKD (Kohara et al., 2017). Although the metabolic disorder in CKD is usually thought to be the cause of the elevated FGF21, its precise mechanism has not been illustrated so far.

\section{FIBROBLAST GROWTH FACTOR 21 AS A POTENTIAL THERAPEUTIC TARGET}

FGF21 has been clarified to have the effect on lowering blood sugar and lipids, so it is expected to be a potential candidate for development of CKD therapeutic. However, short half-life and poor stability are the bottleneck of clinical application of natural FGF21 protein. By constructing a stable mutant FGF21 (mFGF21) and then genetically fusing it with human albumin through a polypeptide to form HSA-mFGF21, whose half-life is 20 times higher than that of FGF21. It can enter the body to play a continuous inhibitory effect on blood glucose, which is expected to become a new biological therapy for metabolic disorders including diabetes (Watanabe et al., 2020). In addition, the FGF21 analog LY2405319 (LY) with the half-life improving exerts an inhibitory effect on blood sugar and lipids, which indicates that the FGF21 pathway may be an ideal candidate for the treatment of metabolic diseases (Adams et al., 2013). The new

\section{REFERENCES}

Adams, A. C., Halstead, C. A., Hansen, B. C., Irizarry, A. R., Martin, J. A., Myers, S. R., et al. (2013). LY2405319, an engineered FGF21 variant. PLoS One 8:e65763. doi: 10.1371/journal.pone.0065763

An, H. J., Lee, B., Kim, D. H., Lee, E. K., Chung, K. W., Park, M. H., et al. (2017). Physiological characterization of a novel PPAR pan agonist, 2-(4-(5,6methylenedioxybenzo[d]thiazol-2-yl)-2-methylphenoxy)-2-methylpropanoic acid (MHY2013). Oncotarget 8, 16912-16924. doi: 10.18632/oncotarget.14818

Ángel, G. M., Paola, V. V., Froylan David, M. S., Lucía, P. B., Juan Mauricio, V. Z., María Fernanda, N. F., et al. (2021). Fibroblast growth factor 21 is associated with increased serum total antioxidant capacity and oxidized lipoproteins in humans with different stages of chronic kidney disease. Ther. Adv. Endocrinol. Metab. 12:20420188211001160. long-acting FGF21 analog PF-05231023 is a promising potential drug for the treatment of type 2 diabetes, obesity and obesityrelated diseases (Weng et al., 2015; Thompson et al., 2016). The current research on targeting FGF21 therapy in CKD has certain limitations with a lack of corresponding clinical trials. At the same time, the current research results of some animal models may not be applicable to humans.

\section{CONCLUSION}

FGF21 signaling and the potential biological role of FGF21 in different types of tissue are summarized and displayed by the schematic diagram (Figure 3). FGF21 regulation performs the potential biological roles in different tissues based on FGFR- $\beta$ klotho signaling transduction, including participating in the glucose and lipid regulation, the improvement of hepatic steatosis, and anti-fibrotic effects in liver, improving damage and fibrosis in kidney, preventing ROS, cardiac hypertrophy, and atherosclerosis in heart, predicting mitochondrial disease in muscles, regulating insulin production in pancreas, and lipid regulation in adipose tissue. The increase of FGF21 levels in CKD patients is influenced by a number of factors, and the pathophysiological significance and its positive or negative impact on patients have not been fully determined. It is possible to speculate that the level of FGF21 is adaptively increased in the early stages of $\mathrm{CKD}$, which contributes to alleviate the metabolic disorders. As the severity of the continuous progress of CKD, the level of FGF21 is also rising correspondingly without more active role. Later the chronically elevated FGF21 may have adverse consequences for the patients with CKD. Consequently, FGF21 may become a potential target and blocking the effect of FGF21$\beta$ klotho endocrine axis may improve the curative effects on CKD patients. However, this needs more researches and clinical trials to further confirm.

\section{AUTHOR CONTRIBUTIONS}

$\mathrm{XZ}$ and NW contributed to the conception and design of the review article. $\mathrm{XZ}$ and $\mathrm{YZ}$ prepared the manuscript. NW revised the manuscript. All authors approved the final draft of the manuscript.

Angelin, B., Larsson, T. E., and Rudling, M. (2012). Circulating fibroblast growth factors as metabolic regulators-a critical appraisal. Cell Metab. 16, 693-705. doi: 10.1016/j.cmet.2012.11.001

Chang, L. H., Hwu, C. M., Chu, C. H., Lin, Y. C., Huang, C. C., You, J. Y., et al. (2021). The combination of soluble tumor necrosis factor receptor type 1 and fibroblast growth factor 21 exhibits better prediction of renal outcomes in patients with type 2 diabetes mellitus. J. Endocrinol. Invest.

Chen, C., Cheung, B. M., Tso, A. W., Wang, Y., Law, L. S., Ong, K. L., et al. (2011). High plasma level of fibroblast growth factor 21 is an Independent predictor of type 2 diabetes: a 5.4-year population-based prospective study in Chinese subjects. Diabetes Care 34, 2113-2115. doi: 10.2337/dc11-0294

Chen, Q., Ma, J., Yang, X., Li, Q., Lin, Z., and Gong, F. (2020). SIRT1 mediates effects of FGF21 to ameliorate cisplatin-induced acute kidney injury. Front. Pharmacol. 11:241. doi: 10.3389/fphar.2020.00241 
Cheng, Y., Zhang, X., Ma, F., Sun, W., Wang, W., Yu, J., et al. (2020). The role of Akt2 in the protective effect of fenofibrate against diabetic nephropathy. Int. J. Biol. Sci. 16, 553-567. doi: 10.7150/ijbs.40643

Coate, K. C., Hernandez, G., Thorne, C. A., Sun, S., Le, T. D. V., Vale, K., et al. (2017). FGF21 is an exocrine pancreas secretagogue. Cell Metab. 25, 472-480. doi: 10.1016/j.cmet.2016.12.004

Cockwell, P., and Fisher, L. A. (2020). The global burden of chronic kidney disease. Lancet 395, 662-664. doi: 10.1016/s0140-6736(19)32977-0

Crasto, C., Semba, R. D., Sun, K., and Ferrucci, L. (2012). Serum fibroblast growth factor 21 is associated with renal function and chronic kidney disease in community-dwelling adults. J. Am. Geriatr. Soc. 60, 792-793. doi: 10.1111/j. 1532-5415.2011.03879.x

Degirolamo, C., Sabbà, C., and Moschetta, A. (2016). Therapeutic potential of the endocrine fibroblast growth factors FGF19. Nat. Rev. Drug Discov. 15, 51-69. doi: $10.1038 / \mathrm{nrd} .2015 .9$

Di Raimondo, D., Tuttolomondo, A., Musiari, G., Schimmenti, C., D’Angelo, A., Pinto, A., et al. (2016). Are the myokines the mediators of physical activityinduced health benefits. Curr. Pharm. Des. 22, 3622-3647. doi: 10.2174/ 1381612822666160429121934

Ding, X., Boney-Montoya, J., Owen, B. M., Bookout, A. L., Coate, K. C., Mangelsdorf, D. J., et al. (2012). BKlotho is required for fibroblast growth factor 21 effects on growth and metabolism. Cell Metab. 16, 387-393. doi: 10.1016/j.cmet.2012.08.002

El-Saeed, A. M., and El-Mohasseb, G. F. (2017). Circulating fibroblast growth factors 21 and 23 as biomarkers of progression in diabetic nephropathy in type 2 diabetes with normoalbuminuria. Egypt J. Immunol. 24, 93-99.

Emanuelli, B., Vienberg, S. G., Smyth, G., Cheng, C., Stanford, K. I., Arumugam, M., et al. (2014). Interplay between FGF21 and insulin action in the liver regulates metabolism. J. Clin. Invest. 124, 515-527. doi: 10.1172/jci6 7353

Esteghamati, A., Khandan, A., Momeni, A., Behdadnia, A., Ghajar, A., Nikdad, M. S., et al. (2017). Circulating levels of fibroblast growth factor 21 in early-stage diabetic kidney disease. Ir. J. Med. Sci. 186, 785-794. doi: 10.1007/s11845-0171554-7

Fang, H., Ghosh, S., Sims, L., Stone, K. P., Hill, C. M., Spires, D., et al. (2021). FGF21 prevents low protein diet-induced renal inflammation in aged mice. Am. J. Physiol. Renal Physiol. 321, F356-F368.

Fernandes-Freitas, I., and Owen, B. M. (2015). Metabolic roles of endocrine fibroblast growth factors. Curr. Opin. Pharmacol. 25, 30-35. doi: 10.1016/j. coph.2015.09.014

GBD Chronic Kidney Disease Collaboration (2020). Global, regional, and national burden of chronic kidney disease1990-2017: a systematic analysis for the global burden of disease study 2017. Lancet 395, 709-733.

Goetz, R., Beenken, A., Ibrahimi, O. A., Kalinina, J., Olsen, S. K., Eliseenkova, A. V., et al. (2007). Molecular insights into the klotho-dependent, endocrine mode of action of fibroblast growth factor 19 subfamily members. Mol. Cell Biol. 27, 3417-3428. doi: 10.1128/mcb.02249-06

Goetz, R., and Mohammadi, M. (2013). Exploring mechanisms of FGF signalling through the lens of structural biology. Nat. Rev. Mol. Cell Biol. 14, 166-180. doi: $10.1038 / \mathrm{nrm} 3528$

González, E., Díez, J. J., Bajo, M. A., Del Peso, G., Grande, C., Rodríguez, O., et al. (2016). Fibroblast growth factor 21 (FGF-21) in peritoneal dialysis patients: natural history and metabolic implications. PLoS One 11:e0151698. doi: 10. 1371/journal.pone.0151698

Han, S. H., Choi, S. H., Cho, B. J., Lee, Y., Lim, S., Park, Y. J., et al. (2010). Serum fibroblast growth factor-21 concentration is associated with residual renal function and insulin resistance in end-stage renal disease patients receiving long-term peritoneal dialysis. Metabolism 59, 1656-1662. doi: 10.1016/j. metabol.2010.03.018

Harrison, S. A., Ruane, P. J., Freilich, B. L., Neff, G., Patil, R., Behling, C. A., et al. (2021). Efruxifermin in non-alcoholic steatohepatitis: a randomized, doubleblind, placebo-controlled, phase 2a trial. Nat. Med. 27, 1262-1271. doi: 10.1038/ s41591-021-01425-3

He, Y., Li, Y., Wei, Z., Zhang, X., Gao, J., Wang, X., et al. (2018). Pharmacokinetics, tissue distribution, and excretion of FGF-21 following subcutaneous administration in rats. Drug Test Anal. 10, 1061-1069. doi: 10. 1002/dta.2365

Hindricks, J., Ebert, T., Bachmann, A., Kralisch, S., Lössner, U., Kratzsch, J., et al. (2014). Serum levels of fibroblast growth factor-21 are increased in chronic and acute renal dysfunction. Clin. Endocrinol. (Oxf.) 80, 918-924. doi: 10.1111/cen. 12380

Inagaki, T., Dutchak, P., Zhao, G., Ding, X., Gautron, L., Parameswara, V., et al. (2007). Endocrine regulation of the fasting response by PPARalpha-mediated induction of fibroblast growth factor 21. Cell Metab. 5, 415-425. doi: 10.1016/j. cmet.2007.05.003

Itoh, N., and Ornitz, D. M. (2011). Fibroblast growth factors: from molecular evolution to roles in development, metabolism and disease. J. Biochem. 149, 121-130. doi: 10.1093/jb/mvq121

Jian, W. X., Peng, W. H., Jin, J., Chen, X. R., Fang, W. J., Wang, W. X., et al. (2012). Association between serum fibroblast growth factor 21 and diabetic nephropathy. Metabolism 61, 853-859. doi: 10.1016/j.metabol.2011.10.012

Kalantar-Zadeh, K., Jafar, T. H., Nitsch, D., Neuen, B. L., and Perkovic, V. (2021). Chronic kidney disease. Lancet

Kharitonenkov, A., Shiyanova, T. L., Koester, A., Ford, A. M., Micanovic, R., Galbreath, E. J., et al. (2005). FGF-21 as a novel metabolic regulator. J. Clin. Invest. 115, 1627-1635.

Kim, D., Lee, J., Bae, I., Kim, M., Huh, Y., Choi, J., et al. (2021). Preparation, characterization, and pharmacological study of a novel long-acting FGF21 with a potential therapeutic effect in obesity. Biologicals 69, 49-58. doi: 10.1016/j. biologicals.2020.11.005

Kim, H. W., Lee, J. E., Cha, J. J., Hyun, Y. Y., Kim, J. E., Lee, M. H., et al. (2013). Fibroblast growth factor 21 improves insulin resistance and ameliorates renal injury in db/db mice. Endocrinology 154, 3366-3376. doi: 10.1210/en.20122276

Kohara, M., Masuda, T., Shiizaki, K., Akimoto, T., Watanabe, Y., Honma, S., et al. (2017). Association between circulating fibroblast growth factor 21 and mortality in end-stage renal disease. PLoS One 12:e0178971. doi: 10.1371/ journal.pone.0178971

Kondo, Y., Komaba, H., and Fukagawa, M. (2020). Endocrine fibroblast growth factors as potential biomarkers for chronic kidney disease. Expert Rev. Mol. Diagn. 20, 715-724. doi: 10.1080/14737159.2020.1780918

Kuro-o, M. (2012). Klotho and $\beta$ Klotho. Adv. Exp. Med. Biol. 728, 25-40.

Kuro-O, M. (2019b). The Klotho proteins in health and disease. Nat. Rev. Nephrol. 15, 27-44. doi: 10.1038/s41581-018-0078-3

Kuro-O, M. (2019a). Klotho and endocrine fibroblast growth factors: markers of chronic kidney disease progression and cardiovascular complications. Nephrol. Dial. Transplant. 34, 15-21. doi: 10.1093/ndt/gfy126

Lancha, A., Frühbeck, G., and Gómez-Ambrosi, J. (2012). Peripheral signalling involved in energy homeostasis control. Nutr. Res. Rev. 25, 223-248. doi: 10.1017/s0954422412000145

Lee, C. H., Hui, E. Y., Woo, Y. C., Yeung, C. Y., Chow, W. S., Yuen, M. M., et al. (2015). Circulating fibroblast growth factor 21 levels predict progressive kidney disease in subjects with type 2 diabetes and normoalbuminuria. .J Clin. Endocrinol. Metab. 100, 1368-1375. doi: 10.1210/jc.2014-3465

Li, S., Wang, N., Guo, X., Li, J., Zhang, T., Ren, G., et al. (2018b). Fibroblast growth factor 21 regulates glucose metabolism in part by reducing renal glucose reabsorption. Biomed. Pharmacother. 108, 355-366. doi: 10.1016/j.biopha.2018. 09.078

Li, F., Liu, Z., Tang, C., Cai, J., and Dong, Z. (2018a). FGF21 is induced in cisplatin nephrotoxicity to protect against kidney tubular cell injury. FASEB J. 32, 3423-3433. doi: 10.1096/fj.201701316r

Li, X. (2019). The FGF metabolic axis. Front. Med. 13:511-530. doi: 10.1007/ s11684-019-0711-y

Lin, S., Yu, L., Ni, Y., He, L., Weng, X., Lu, X., et al. (2020). fibroblast growth factor 21 attenuates diabetes-induced renal fibrosis by negatively regulating TGF- $\beta$ p53-Smad2/3-mediated epithelial-to-mesenchymal transition via activation of AKT. Diabetes Metab. J. 44, 158-172. doi: 10.4093/dmj.2018.0235

Lin, Z., Pan, X., Wu, F., Ye, D., Zhang, Y., Wang, Y., et al. (2015). Fibroblast growth factor 21 prevents atherosclerosis by suppression of hepatic sterol regulatory element-binding protein-2 and induction of adiponectin in mice. Circulation 131, 1861-1871. doi: 10.1161/circulationaha.115.015308

Lin, Z., Zhou, Z., Liu, Y., Gong, Q., Yan, X., Xiao, J., et al. (2011). Circulating FGF21 levels are progressively increased from the early to end stages of chronic kidney diseases and are associated with renal function in Chinese. PLoS One 6:e18398. doi: 10.1371/journal.pone.0018398

Looker, H. C., Colombo, M., Hess, S., Brosnan, M. J., Farran, B., Dalton, R. N., et al. (2015). Biomarkers of rapid chronic kidney disease progression in type 2 diabetes. Kidney Int. 88, 888-896. doi: 10.1038/ki.2015.199 
Luo, Y., Ye, S., Chen, X., Gong, F., Lu, W., and Li, X. (2017). Rush to the fire: FGF21 extinguishes metabolic stress, metaflammation and tissue damage. Cytokine Growth Factor Rev. 38, 59-65. doi: 10.1016/j.cytogfr.2017.08.001

Marchelek-Myśliwiec, M., Dziedziejko, V., Nowosiad-Magda, M., Dołêgowska, K., Dołêgowska, B., Pawlik, A., et al. (2019). chronic kidney disease is associated with increased plasma levels of fibroblast growth factors 19 and 21. Kidney Blood Press Res. 44, 1207-1218. doi: 10.1159/000502647

Musso, G., Cassader, M., Cohney, S., Pinach, S., Saba, F., and Gambino, R. (2015). Emerging liver-kidney interactions in nonalcoholic fatty liver disease. Trends Mol. Med. 21, 645-662. doi: 10.1016/j.molmed.2015.08.005

Nishimura, T., Nakatake, Y., Konishi, M., and Itoh, N. (2000). Identification of a novel FGF. Biochim. Biophys. Acta. 1492, 203-206. doi: 10.1016/s01674781(00)00067-1

Nohara, S., Ishii, A., Yamamoto, F., Yanagiha, K., Moriyama, T., Tozaka, N., et al. (2019). GDF-15, a mitochondrial disease biomarker, is associated with the severity of multiple sclerosis. J. Neurol. Sci. 405:116429. doi: 10.1016/j.jns.2019. 116429

Packer, M. (2020). Role of ketogenic starvation sensors in mediating the renal protective effects of SGLT2 inhibitors in type 2 diabetes. J. Diabetes Complications 34:107647. doi: 10.1016/j.jdiacomp.2020.107647

Pan, X., Shao, Y., Wu, F., Wang, Y., Xiong, R., Zheng, J., et al. (2018). FGF21 prevents angiotensin II-induced hypertension and vascular dysfunction by activation of ACE2/angiotensin-(1-7) axis in mice. Cell Metab. 27, 1323.e1337.e.

Patel, V. J., Joharapurkar, A. A., Kshirsagar, S. G., Sutariya, B. K., Patel, M. S., Patel, H. M., et al. (2018). Coagonist of glucagon-like peptide-1 and glucagon receptors ameliorates kidney injury in murine models of obesity and diabetes mellitus. World J. Diabetes 9, 80-91. doi: 10.4239/wjd.v9.i6.80

Planavila, A., Redondo, I., Hondares, E., Vinciguerra, M., Munts, C., Iglesias, R., et al. (2013). Fibroblast growth factor 21 protects against cardiac hypertrophy in mice. Nat. Commun. 4:2019.

Planavila, A., Redondo-Angulo, I., Ribas, F., Garrabou, G., Casademont, J., Giralt, M., et al. (2015). Fibroblast growth factor 21 protects the heart from oxidative stress. Cardiovasc. Res. 106, 19-31. doi: 10.1093/cvr/cvu263

Potthoff, M. J., Inagaki, T., Satapati, S., Ding, X., He, T., Goetz, R., et al. (2009). FGF21 induces PGC-1alpha and regulates carbohydrate and fatty acid metabolism during the adaptive starvation response. Proc. Natl. Acad. Sci. U.S.A. 106, 10853-10858. doi: 10.1073/pnas.0904187106

Reitman, M. L. (2013). FGF21 mimetic shows therapeutic promise. Cell Metab. 18, 307-309. doi: 10.1016/j.cmet.2013.08.014

Shi, Y. C., Lu, W. W., Hou, Y. L., Fu, K., Gan, F., Cheng, S. J., et al. (2018). protection effect of exogenous fibroblast growth factor 21 on the kidney injury in vascular calcification rats. Chin. Med. J. (Engl.) 131, 532-538. doi: 10.4103/ 0366-6999.226065

Silverwood, R. J., Pierce, M., Thomas, C., Hardy, R., Ferro, C., Sattar, N., et al. (2013). Association between younger age when first overweight and increased risk for CKD. J. Am. Soc. Nephrol. 24, 813-821. doi: 10.1681/asn.2012070675

Stein, S., Bachmann, A., Lössner, U., Kratzsch, J., Blüher, M., Stumvoll, M., et al. (2009). Serum levels of the adipokine FGF21 depend on renal function. Diabetes Care 32, 126-128. doi: 10.2337/dc08-1054

Suassuna, P. G. A., Cherem, P. M., de Castro, B. B., Maquigussa, E., Cenedeze, M. A., Lovisi, J. C. M., et al. (2020). $\alpha$ Klotho attenuates cardiac hypertrophy and increases myocardial fibroblast growth factor 21 expression in uremic rats. Exp. Biol. Med. (Maywood) 245, 66-78. doi: 10.1177/1535370219894302

Suomalainen, A. (2013). Fibroblast growth factor 21: a novel biomarker for human muscle-manifesting mitochondrial disorders. Expert Opin. Med. Diagn. 7, 313317. doi: 10.1517/17530059.2013.812070

Suomalainen, A., Elo, J. M., Pietiläinen, K. H., Hakonen, A. H., Sevastianova, K., Korpela, M., et al. (2011). FGF-21 as a biomarker for muscle-manifesting mitochondrial respiratory chain deficiencies: a diagnostic study. Lancet Neurol. 10, 806-818. doi: 10.1016/s1474-4422(11)70155-7

Tanimura, Y., Aoi, W., Takanami, Y., Kawai, Y., Mizushima, K., Naito, Y., et al. (2016). Acute exercise increases fibroblast growth factor 21 in metabolic organs and circulation. Physiol. Rep. 4:e12828.

Thompson, W. C., Zhou, Y., Talukdar, S., and Musante, C. J. (2016). PF-05231023, a long-acting FGF21 analogue, decreases body weight by reduction of food intake in non-human primates. J. Pharmacokinet. Pharmacodyn. 43, 411-425. doi: 10.1007/s10928-016-9481-1

Wang, Y. S., Ye, J., Cao, Y. H., Zhang, R., Liu, Y., Zhang, S. W., et al. (2019). Increased serum/plasma fibroblast growth factor 21 in type 2 diabetes mellitus: a systematic review and meta-analysis. Postgrad. Med. J. 95, 134-139. doi: 10.1136/postgradmedj-2018-136002

Watanabe, H., Miyahisa, M., Chikamatsu, M., Nishida, K., Minayoshi, Y., Takano, M., et al. (2020). Development of a long acting FGF21 analogue-albumin fusion protein and its anti-diabetic effects. J. Control. Release 324, 522-531.

Wei, W., An, X. R., Jin, S. J., Li, X. X., and Xu, M. (2018). Inhibition of insulin resistance by PGE1 via autophagy-dependent FGF21 pathway in diabetic nephropathy. Sci. Rep. 8:9.

Weng, Y., Chabot, J. R., Bernardo, B., Yan, Q., Zhu, Y., Brenner, M. B., et al. (2015). Pharmacokinetics (PK), pharmacodynamics (PD) and integrated PK/PD modeling of a novel long acting FGF21 clinical candidate PF-05231023 in diet-induced obese and leptin-deficient obese mice. PLoS One 10:e0119104. doi: 10.1371/journal.pone.0119104

Woo, Y. C., Xu, A., Wang, Y., and Lam, K. S. (2013). Fibroblast growth factor 21 as an emerging metabolic regulator: clinical perspectives. Clin. Endocrinol. (Oxf.) 78, 489-496. doi: 10.1111/cen.12095

Wu, C. H., Chou, R. H., Kuo, C. S., Huang, P. H., Chang, C. C., Leu, H. B., et al. (2018). Circulating fibroblast growth factor 21 is associated with subsequent renal injury events in patients undergoing coronary angiography. Sci. Rep. $8: 12425$.

Yamamoto, S., Koyama, D., Igarashi, R., Maki, T., Mizuno, H., Furukawa, Y., et al. (2020). Serum endocrine fibroblast growth factors as potential biomarkers for chronic kidney disease and various metabolic dysfunctions in aged patients. Intern. Med. 59, 345-355. doi: 10.2169/internalmedicine.3597-19

Yu, W., Zhu, H., Chen, X., Gu, X., Zhang, X., Shen, F., et al. (2019). Genetic variants flanking the FGF21 gene were associated with renal function in chinese patients with type 2 diabetes. J Diabetes Res. 2019:9387358.

Zhang, C., Shao, M., Yang, H., Chen, L., Yu, L., Cong, W., et al. (2013). Attenuation of hyperlipidemia- and diabetes-induced early-stage apoptosis and late-stage renal dysfunction via administration of fibroblast growth factor-21 is associated with suppression of renal inflammation. PLoS One 8:e82275. doi: 10.1371/ journal.pone.0082275

Zhang, F., Yu, L., Lin, X., Cheng, P., He, L., Li, X., et al. (2015). Minireview: roles of fibroblast growth factors 19 and 21 in metabolic regulation and chronic diseases. Mol. Endocrinol. 29, 1400-1413. doi: 10.1210/me.2015-1155

Zhang, J., and Li, Y. (2015). Fibroblast growth factor 21 analogs for treating metabolic disorders. Front. Endocrinol. (Lausanne) 6:168. doi: 10.3389/fendo. 2015.00168

Zhang, R., Cai, X., Du, Y., Li, W., Liu, L., and Lin, Q. (2021). Association of serum fibroblast growth factor 21 and urinary glucose excretion in hospitalized patients with type 2 diabetes. J. Diabetes Complications 35:107750. doi: 10.1016/ j.jdiacomp.2020.107750

Zhang, Y., Liu, Z., Zhou, M., and Liu, C. (2018). Therapeutic effects of fibroblast growth factor-21 against atherosclerosis via the NF- $\kappa$ B pathway. Mol. Med. Rep. $17,1453-1460$.

Zhao, L., Wang, H., Xie, J., Chen, Z., Li, X., and Niu, J. (2017). Potent long-acting rhFGF21 analog for treatment of diabetic nephropathy in $\mathrm{db} / \mathrm{db}$ and DIO mice. BMC Biotechnol. 17:58. doi: 10.1186/s12896-0170368-z

Zhu, L., Li, M., Zha, Q., Yang, M., Yu, J., Pan, M., et al. (2021). Fibroblast growth factor 21 (FGF21) is a sensitive marker of osteoporosis in haemodialysis patients: a cross-sectional observational study. BMC Nephrol. 22:183. doi: 10. 1186/s12882-021-02393-z

Conflict of Interest: The authors declare that the research was conducted in the absence of any commercial or financial relationships that could be construed as a potential conflict of interest.

Publisher's Note: All claims expressed in this article are solely those of the authors and do not necessarily represent those of their affiliated organizations, or those of the publisher, the editors and the reviewers. Any product that may be evaluated in this article, or claim that may be made by its manufacturer, is not guaranteed or endorsed by the publisher.

Copyright (c) 2021 Zhou, Zhang and Wang. This is an open-access article distributed under the terms of the Creative Commons Attribution License (CC BY). The use, distribution or reproduction in other forums is permitted, provided the original author(s) and the copyright owner(s) are credited and that the original publication in this journal is cited, in accordance with accepted academic practice. No use, distribution or reproduction is permitted which does not comply with these terms. 